\title{
Multi-Variable Intelligent Matching Pursuit Algorithm Using Prior Knowledge for Image Reconstruction by $l_{0}$ Minimization
}

\author{
Dan Li, Qiang Wang Member IEEE, Yi Shen Member IEEE ${ }^{1}$ \\ Control Science and Engineering, Harbin Institute of Technology, \\ No.92, West Da-Zhi Street, Nangang District, Harbin, China, 150001 \\ lidanhit@163.com,wangqiang@hit.edu.cn, shen@hit.edu.cn
}

\begin{abstract}
Image reconstruction by $l_{0}$ minimization is an NP-hard problem that requires exhaustively listing all possibilities of the original signal with a very high computational complexity, which is difficulty to be achieved by traditional algorithms. Although greedy algorithm aims at solving $l_{0}$ minimization, it is more likely to fall into a suboptimal solution. In this paper, we propose a multi-variable intelligent matching pursuit algorithm (MIMP), which can solve $l_{0}$ minimization problem essentially by taking the advantage of the intelligent optimization algorithm in solving combinatorial optimization problems and searching for the global optimal solution to improve the performance of image reconstruction. The updating mechanism of MIMP is designed by introducing the matching strategies of greedy algorithm to accelerate the reconstruction speed. Also, the multi-variable scheme is utilized to sample images and then the joint reconstruction is implemented to the measurements, which can not only improve the reconstruction accuracy but also reduce the computational complexity. Moreover, the edge saliency can be obtained as the prior knowledge to guide the compressive sensing reconstruction, which contributes a lot to reduce the computational complexity and accelerate the reconstruction speed. As the sparsity level of image is hard to be estimated, a new optimization function is proposed to solve this problem without knowing the sparsity level as a prior. Compared with other state-of-the-art algorithms, the proposed method MIMP can achieve
\end{abstract}


a better reconstruction accuracy by solving $l_{0}$ minimization essentially with intelligent optimization algorithms. Also, MIMP has a reasonable relatively faster reconstruction speed by introducing the matching strategies of greedy algorithm and using the edge saliency as a prior knowledge. Numerical experiments on several images demonstrate that the proposed method MIMP significantly outperforms the state-of-the-art algorithms and the structure based reconstruction algorithms in PSNR, SSIM and visual quality.

Keywords: Compressive sensing, $l_{0}$ minimization, Intelligent optimization algorithm, Prior knowledge, Multi-variable scheme.

\section{Introduction}

Compressive sensing (CS) [1, 2, 3] theory, proposed by Candes and Donoho in 2006, shows that signal can be reconstructed from many fewer measurements than suggested by Nyquist sampling theory if the signal is sparse or compressive 5 in some domains. If the original signal $x \in R^{N * 1}$ is compressive, it can be represented sparsely in the sparse basis $\psi$. The measurement signal $y \in R^{M * 1}$ $(M<N)$ is obtained by $y=\phi * x$, where $\phi$ is uncorrelated to $\psi$ and satisfies restrict isometry property (RIP). The relations can be represented by Equ.(1).

$$
y=\phi * x=\phi * \psi * s
$$

where, $s$ is the sparse representation of $x$ in the sparse basis $\psi$.

To simplify Equ.(1), $x$ is assumed to be sparse in the following sections. As we all know, reconstruction is a process of recovering high-dimensional signal $x$ from low-dimensional signal $y$, which can be obtained by optimizing a constrained optimization problem of sparsity. Then the constrained optimization problem of sparsity can be implemented by the $l_{0}$ minimization problem, which 15 can be described in Equ.(2).

$$
x=\operatorname{argmin}\|x\|_{0} \quad \text { subject to } \quad y=\phi * x,
$$


where, $x$ represents the $l_{0}$ norm of $x$, which is defined by the number of nonzero elements of $x$. Equ.(2) can be also written as the form of Equ.(3).

$$
x=\operatorname{argmin}\|x\|_{0}+\lambda *\|y-\phi * x\|_{2}^{2},
$$

where $\lambda$ is a positive constant between 0 and 1 , which used to balance the relative weight between the sparsity and the data fidelity item.

However, $l_{0}$ minimization is an NP-hard problem that requires exhaustively listing all possibilities of the original signal and is difficult to be achieved by traditional algorithms. Since $l_{1}$ norm [4] is the optimal convex approximation of $l_{0}$ norm and it has been proved that $l_{1}$ norm is equivalent to the $l_{0}$ norm for many problems. It is widely adopted that the $l_{0}$ minimization probelm can be solved by relaxing it to the $l_{1}$ minimization problem [5, 6, 7] which is shown in Equ.(4). Nevertheless, a fact that is often neglected is, the conditions guaranteeing the equivalence of $l_{0}$ minimization and $l_{1}$ minimization are not necessarily satisfied, which leads to a suboptimal solution. Many reconstruction methods have been already proposed recently, such as gradient projection sparse 30 reconstruction [8, 9], non-linear filter [10] and iterative threshold [11], which also transform $l_{0}$ minimization to other mathematical models and are more likely to fall into suboptimal solutions.

$$
x=\operatorname{argmin}\|x\|_{1}+\lambda *\|y-\phi * x\|_{2}^{2} .
$$

The methods which aims at solving $l_{0}$ minimization problem available for compressive sensing reconstruction fall into two categories: greedy algorithm [12, 13, 14] and heuristic method. The objective of greedy algorithm is to estimate the support collection and its corresponding coefficients by iterating the coefficients based on the residual correlation. However, the residual correlation can only reflect the correlation degree between coefficients and the measurement signal, but can't indicate that the bigger the residual correlation is, the

40 bigger the nonzero coefficient is. Also, greedy algorithm is more likely to find a suboptimal solution and can not solve $l_{0}$ minimization essentially. Intelligen- 
t optimization algorithm [15, 16] is a class of heuristic optimization methods which is famous for its global searching ability and superior performance in solving combinatorial optimization problems. It is natural to consider that in-

45 telligent optimization algorithm can be used to solve $l_{0}$ minimization problem. Two methods [17, 18] based on simulated annealing algorithm are proposed for CS reconstruction by $l_{0}$ minimization. However, the single-cycle local searching scheme in 17, 18] is hard to find the global optimal solution. Also, the precondition of [17, 18] is that the sparsity level must be known, which is a very strong

${ }_{50}$ limitation as the sparsity level is always unknown in practice. In our precious work [19, 20], we use genetic algorithm and artificial immune algorithm [21, 22] to solve $l_{0}$ minimization directly. However, due to the randomness of intelligent optimization algorithm, the computational complexity of the proposed methods in [19, 20] is high and the reconstruction speed of them slow down.

Image reconstruction [23, 24, 25], which is a fundamental problem in the field of image processing, aims to reconstruct the original image with high quality from the degraded measurements. Prior knowledge [26, 27] plays an importan$\mathrm{t}$ role in image reconstruction and it has been proved that introducing prior knowledge can improve the efficiency of image reconstruction algorithms. One

60 way of improving the reconstruction quality is to exploit the co-called structured sparsity. Some methods using particular structures such as tree structure [28, 29, 30] and dependence structure [31] of the wavelet coefficients have been applied in image reconstruction. Tree-structured correlations of wavelet coefficients is utilized to propose a group-sparsity regulation method in [32]. Bayesian

65 CS reconstruction methods exploiting the prior of the wavelet tree structures have also been developed in [33, 34, 35]. Under the wavelet transformation, the main energy of image is concentrated on the wavelet coefficients of the lowfrequency subspace and the wavelet coefficients of the high-frequency subspace show high sparsity, which means that the wavelet coefficients corresponding to the edge are significantly different from zero. Suppose the original image is locally smooth except edge area, the locations of the nonzero wavelet coefficients of the high-frequency parts happen to correspond with the edge of the original 
image. So if the edge information of the image is extracted, it can be applied as prior knowledge to guide the process of image reconstruction, which can reduce the computational complexity. Several representative works based on the guidance of edge information have been proposed, such as edge guided MRI reconstruction [36] and edge based multiple pursuit algorithm (EMPA) 37]. Moreover, a multi-variable scheme using conventional linear sampling and compressive sampling for low frequency coefficients and high frequency coefficients 80 respectively is developed for image reconstruction in [38].

In this paper, we propose a multi-variable intelligent pursuit algorithm (MIMP) using prior knowledge to solve $l_{0}$ minimization problem essentially. MIMP aims at searching the support collection and its corresponding coefficients of the original signal intelligently and accurately. As the sparsity level of image ${ }_{85}$ is hard to be estimated, a new optimization function is proposed when the sparsity level is unknown in advance. Due to the superior global optimizing strategy of intelligent optimization algorithm, artificial immune algorithm (AIA) is introduced to guide the process of support collection estimation. Artificial immune algorithm performs well in both global searching and local searching, which makes MIMP more likely to find the global optimal solution and have a fast reconstruction speed. The principle of the intelligent estimation is designed by the matching strategies of greedy algorithm, which is beneficial to reduce the computational complexity. Also, based on the high sparsity of the highfrequency parts of wavelet transformation, the multiple measurement scheme, which uses conventional linear sampling for the low-frequency coefficients and compressive sensing for the high-frequency coefficients, is introduced into MIMP to improve the reconstruction quality. In addition, as the edge saliency mostly happens to correspond with the locations of the nonzero wavelet coefficients in high-frequency subspace, it is introduced into MIMP to guide the process of global searching, which can reduce the computational complexity. As MIMP can solve $l_{0}$ minimization essentially and by means of the combination of edge saliency and multi-variable joint reconstruction, the proposed method MIMP significantly improves the reconstruction quality of those images with the ob- 
vious edges and high sparsity, such as computed tomography (CT) images and magnetic resonance (MR) images. Experiments on image reconstruction demonstrate that the proposed method MIMP produces more superior reconstruction performance than other state-of-the-art algorithms, such as multi-variable OMP (M-OMP), multi-variable Polytope Faces Pursuit algorithm (M-PFP) [39], multi-variable backtracking-based matching pursuit (M-BAOMP) [40], multivariable compressive sampling orthogonal matching pursuit (M-CoSaOMP) 41], multi-variable Sparsity Adaptive Matching Pursuit algoritm (M-SAMP) [42], tree-based CoSaMP (Tree-CoSaMP) [43], tree-structured wavelet compressive sening (TSWCS) [33] and edge based multiple pursuit algorithm (EMPA), edge based compressive sensing algorithm (EdgeCS), multi-variable pursuit algorith$115 \mathrm{~m}$ using multi-variable $\mathrm{K}$ distribution (MPA-MK) and multi-variable pursuit algorithm using multi-variable Laplace distribution (MPA-ML).

The major contributions of this paper are fourfold:

1. MIMP improves the reconstruction quality significantly by taking the advantage of intelligent optimization algorithm in solving $l_{0}$ minimization essentially and accelerates the reconstruction speed by introducing matching strategies of greedy algorithm.

2. As the sparsity level of image is hard to be estimated, a new optimization function is proposed for CS reconstruction when the sparsity level is unknown in advance.

3. A multi-variable scheme, including multiple measurement and multi-variable joint reconstruction, is introduced to improve the performance of image reconstruction significantly with fewer measurements.

4. The edge saliency is applied as prior knowledge to guide the process of image reconstruction, which can reduce the computational complexity and improve the reconstruction quality, especially for images with obvious edges and high sparsity in wavelet domain.

The layout of this paper is organized as follows: In section 2, the proposed 
framework MIMP for image reconstruction based on $l_{0}$ minimization is provided. Experiment results are given in Section 3 to illustrate the performance of the proposed method MIMP. Section 4 concludes this paper.

The follow notations are used in the rest of this paper. The $l_{0}$ norm of signal $x$ can be represented by $\|x\|_{0}$. Let $\phi$ indicate the measurement matrix and $\phi_{i}$ represents the ith column of $\phi . S=1,2, \ldots, N$ indicates the index of each entry of the original signal, where $N$ represents the length of the original signal. Suppose $m$ entries are selected from $S$ to generate the support collection $I$, the vector $x_{I}$ consists the entries of $x$ indexed by $i \subset I$ and the matrix $\phi_{I}$ is composed of the columns of $\phi$ with indexes $i \subset I$. The pseudo-inverse operation of measurement matrix $\phi$ can be calculated by $\phi^{\dagger}=\left(\phi^{\top} * \phi\right)^{-1} * \phi^{\top}$, where $T$ represents matrix transportation. The subscript ${ }_{L L}$ represents the lowfrequency part of wavelet transformation and the subscripts $H L, L H$ and $H H$ represent the high-frequency parts respectively.

\section{Method MIMP}

In this section, we first introduce the multi-variable scheme and the prior knowledge. Then the diagram of the proposed method MIMP is described in detail. In the end, we analysis the computational complexity.

\subsection{Multi-variable scheme}

The multi-variable scheme includes two parts, i.e. multiple measurement and multi-variable joint reconstruction. The schematic diagram of multi-variable scheme and an example to describe the process of multiple measurement is shown in Figure 1.

The multiple measurement scheme is based on the multiscale decomposition by using conventional linear sampling for low-frequency wavelet coefficients and compressive measurement on high-frequency wavelet coefficients. This framework of measurement can extract more useful prior knowledge for compressive sensing reconstruction. Suppose the original image is $X$. Firstly, the original 


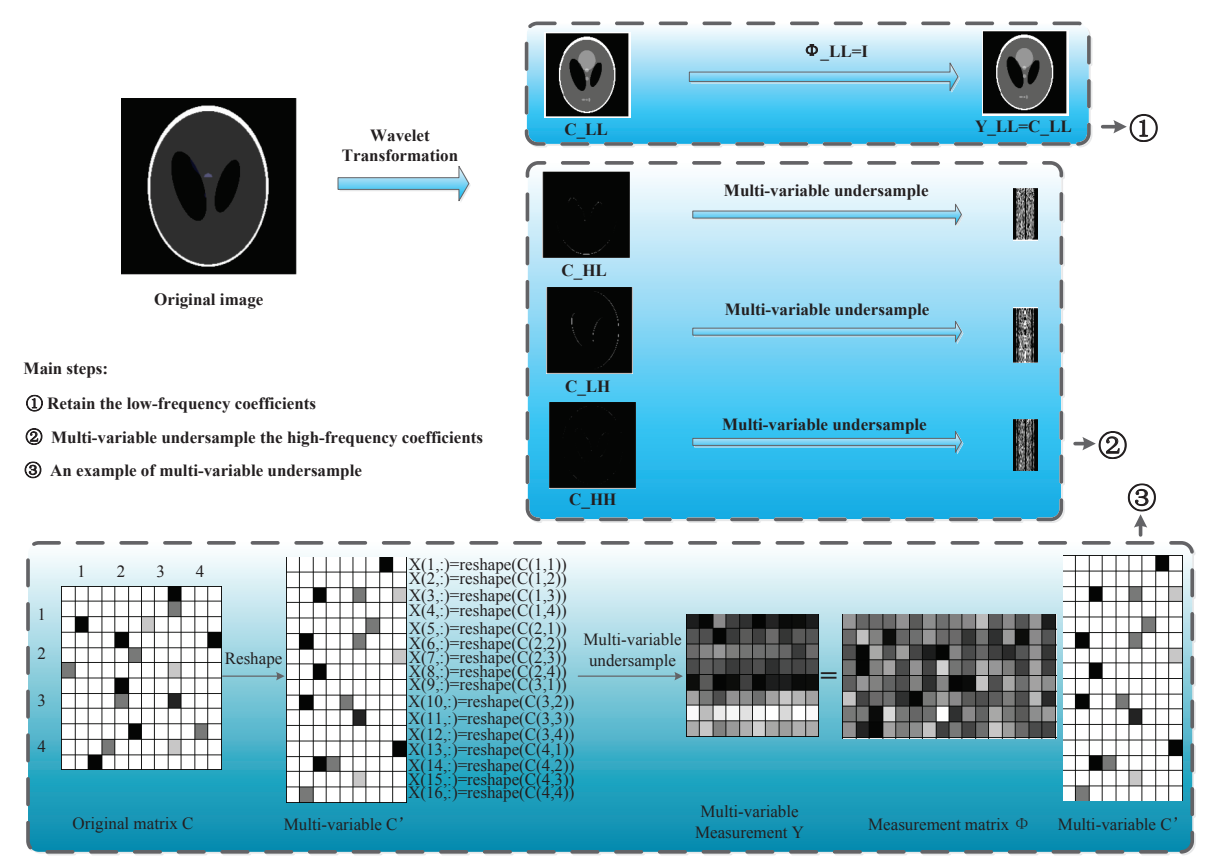

Figure 1: The schematic diagram of multi-variable scheme. The main step 3 is an example of multiple measurement for the high frequency coefficients. The size of the original matrix $C$ is $12 * 12$ and the size of neighbour is set as $3 * 3$. Each neighbour can be reshaped to a signal with size of $1 * 9$ and $C$ is represented as a $16 * 9$ multi-variable matrix $C^{\prime}$. The $8 * 9$ measurement image $Y$ can be obtained by a Gaussian random matrix $\Phi$ with size of $8 * 16$.

image is decomposed by one scale wavelet decomposition to obtain the wavelet coefficients in each scale, i.e. $C_{L L}, C_{L H}, C_{H L}$ and $C_{H H}$. After the wavelet decomposition, low-frequency coefficients $C_{L L}$ is measured using the conventional linear measurement to retain the whole $C_{L L}$ in the encoding phase, and the high-frequency coefficients $C_{L H}, C_{H L}$ and $C_{H H}$ are down-sampled by a Gaussian random matrix. As we all know, the wavelet coefficients hold the property of aggregation, which indicates that if a wavelet coefficient is large, the coefficients adjacent to it are also very large, and vice versa. This property makes the neighbored wavelet coefficients much correlated and dependent. So when down-sample the high frequency coefficients, we sample the wavelet coefficients neighbors instead of all the wavelet coefficients with Gaussian random matrix, which transforms the unit-variable measurement to a multiple measurement. 
Given the size of wavelet coefficients neighbors $d * d, C_{L H}\left(C_{H L}, C_{H H}\right)$ is reshaped to a multi-variable form $C_{L H}^{\prime}\left(C_{H L}^{\prime}, C_{H H}^{\prime}\right)$ with size of $N^{\prime} * d^{2}$, where $N^{\prime}=N / d$. The multi-variable measurement matrix $Y$ can be obtained by a measurement matrix with size of $M * N^{\prime}$. In particularly, $C_{L H}^{\prime}\left(C_{H L}^{\prime}, C_{H H}^{\prime}\right)$ and $Y$ will be reduced as a signal $x$ with size of $N^{\prime} * 1$ and $y$ with size of $M^{\prime} * 1$ when $d=1$, which converts back to the unit-variable reconstruction.

After multiple measurement, $X^{\prime}$ is a joint sparse matrix, which can be jointly reconstructed from the measurement image $Y$ and the measurement matrix $\Phi$. The formulation of multi-variable joint reconstruction can be represented by Equ.(5).

$$
\min _{X^{\prime}} \operatorname{supp}\left(X^{\prime}\right) \quad \text { subject to } \quad Y=\Phi * X^{\prime}
$$

where $\operatorname{supp}\left(X^{\prime}\right)=\sum_{j}^{N} \operatorname{supp}\left(X^{\prime}(:, j)\right), X^{\prime}(:, j)$ represents the $j$ th column of $X^{\prime}$ and $M$ represents the number of measurements.

\subsection{Prior knowledge}

Under the framework of multi-variable scheme, we can obtain the whole low-frequency wavelet coefficients and the down-sampled high-frequency wavelet coefficients in the encoding phase of compressive sensing framework. Since most energy of image is concentrated on the low-frequency wavelet coefficients, we can extract the edge saliency using the whole low-frequency coefficients as the prior knowledge. Firstly, set $C_{H L}^{\prime}=0, C_{L H}^{\prime}=0, C_{H H}^{\prime}=0$ and implement inverse wavelet transformation for $C_{L H}^{\prime}, C_{H L}^{\prime}, C_{H H}^{\prime}$ and $C_{L L}$ to obtain a lowfrequency image $X_{l}$. Secondly, extract the edge of the low-frequency image using edge detection algorithm, such as canny or sobel. Thirdly, implement wavelet decomposition to the extracted edge to obtain the wavelet coefficients $e d g e_{H L}$, $e d g e_{L H}$ and $e d g e_{H H}$ in high-frequency subspace which are mostly consistent with the locations of nonzero wavelet coefficients in $C_{H L}, C_{L H}$ and $C_{H H}$. The $e^{e d g e_{H L}}, e d g e_{L H}$ and $e d g e_{H H}$ are sampled based on the multiple measurement scheme shown in Fig.1. The coefficients corresponding to the edge information 
are named as edge coefficients and the others, which are very small or equal to zero, are named as non-edge coefficients. In the multi-variable form, neighbors which include edge coefficients are edge neighbors and that do not contain edge coefficients are non-edge neighbors. Let $E$ represent the set of indexes of edge neighbors and $E N$ represent the set of indexes of non-edge neighbors.

\subsection{MIMP for image reconstruction}

For the problem of multi-variable reconstruction, each column of $X^{\prime}$ can be represented as a signal $x$ and reconstructed through the corresponding column of $Y$ and the measurement matrix $\Phi$. The proposed method MIMP aims at estimating the support collection $I$ and its corresponding coefficients of the original signal intelligently and accurately. If the sparsity level $K$ is known, for the support collection $I$, its corresponding coefficients can be calculated by Equ.(6) and the reconstructed signal recx can be obtained by Equ.(7).

$$
\begin{gathered}
\operatorname{rec}_{I}=\Phi_{I}^{\dagger} * y \\
\operatorname{rec}_{I}=\Phi_{I}^{\dagger} * y \quad \text { and } \operatorname{rec}_{S-I}=0 .
\end{gathered}
$$

If the support collection $I$ can be estimated, it must satisfy $\left\|\Phi * \Phi_{I}^{\dagger} * y-y\right\|_{2}^{2}=$ 0 . As $\left\|\Phi * \Phi_{I}^{\dagger} * y-y\right\|_{2}^{2} \geq 0$, the reconstruction problem can be transformed to 215 optimize the optimization model Equ.(8).

$$
\min _{I} f(I)=\left\|\Phi_{I} * \Phi_{I}^{\dagger} * y-y\right\|_{2}
$$

As mentioned above, the optimization model described in Equ.(8) requires a precondition that the sparsity level $K$ is known in advance. However, the sparsity level of image is hard to be estimated in practice. Aiming at this problem, we propose a new optimization model when the sparsity level is unknown in advance. Define the measurement collection $I_{m m}$, which represents the collection including $\mathrm{mm}$ (where $\mathrm{mm}$ is larger than $K$ and smaller than the measurement 
number $M$ ) indexes selected in $S . I_{m m}$ can also be used to reconstruct the original signal through Theorem 1 proposed in this paper.

Theorem 1: if the sparsity level is unknown in advance, the measurement 225 collection $I_{m m}$, which contains the support collection of the original signal, can be estimated by optimizing the optimization model described in Equ.(9) and the reconstructed signal can be obtained by Equ.(10).

$$
\begin{gathered}
f\left(I_{m m}\right)=\left\|\Phi_{I_{m m}} * \Phi_{I_{m m}}^{\dagger} * y-y\right\|_{2}, \\
\operatorname{recx}_{I_{m m}}=\Phi_{I_{m m}}^{\dagger} * y \quad \text { and } \quad r e c_{S-I_{m m}}=0 .
\end{gathered}
$$

Proof: Suppose $\Phi$ is the measurement matrix and the columns of $\Phi$ are in general position, $I_{0}$ denotes the support of the original signal $x_{0}$ and $I_{s}$ denotes the support of $x_{s}$, if $I_{0}$ is a subset of $I_{s}$, then $x_{s}=x_{0}$. As the support collection $I$ satisfies $\left\|\Phi_{I} * \Phi_{I}^{\dagger} * y-y\right\|_{2}=0$, if the measurement collection $I_{m m}$ includes $I, I_{m m}$ must satisfy the condition that $\left\|\Phi_{I_{m m}} * \Phi_{I_{m m}}^{\dagger} * y-y\right\|_{2}=0$. So Equ.(9) can be set as the objective function when the sparsity level is unknown and the reconstructed signal can be obtained by Equ.(10).

Artificial immune algorithm (AIA) is an intelligent optimization algorithm which is achieved by simulating the process of immune system. AIA performs better not only in global searching but also in local searching. In AIA, all the possible solutions consist of the searching area and each individual represents a possible solution $I_{m m}$. After the process of AIA, the obtained memorycell can be set as the estimated $I_{m m}$. The procedure of MIMP used for image reconstruction is shown in Algorithm 1. Moreover, we explain the details of MIMP clearly with the following steps.

(1) Fitness function. As shown in Theorem 1, the proposed optimization function $f\left(I_{m m}\right)=\left\|\Phi_{I_{m m}} * \Phi_{I_{m m}}^{\dagger} * y-y\right\|_{2}^{2}$ is set as the fitness function of AIA.

(2) Initialization. As mentioned in Section 2.2, the edge coefficients are mostly consistent with the locations of nonzero elements in $C_{H L}, C_{L H}$ and $C_{H H}$, so we select $m m$ indexes from $E$ to generate the initial individuals. 


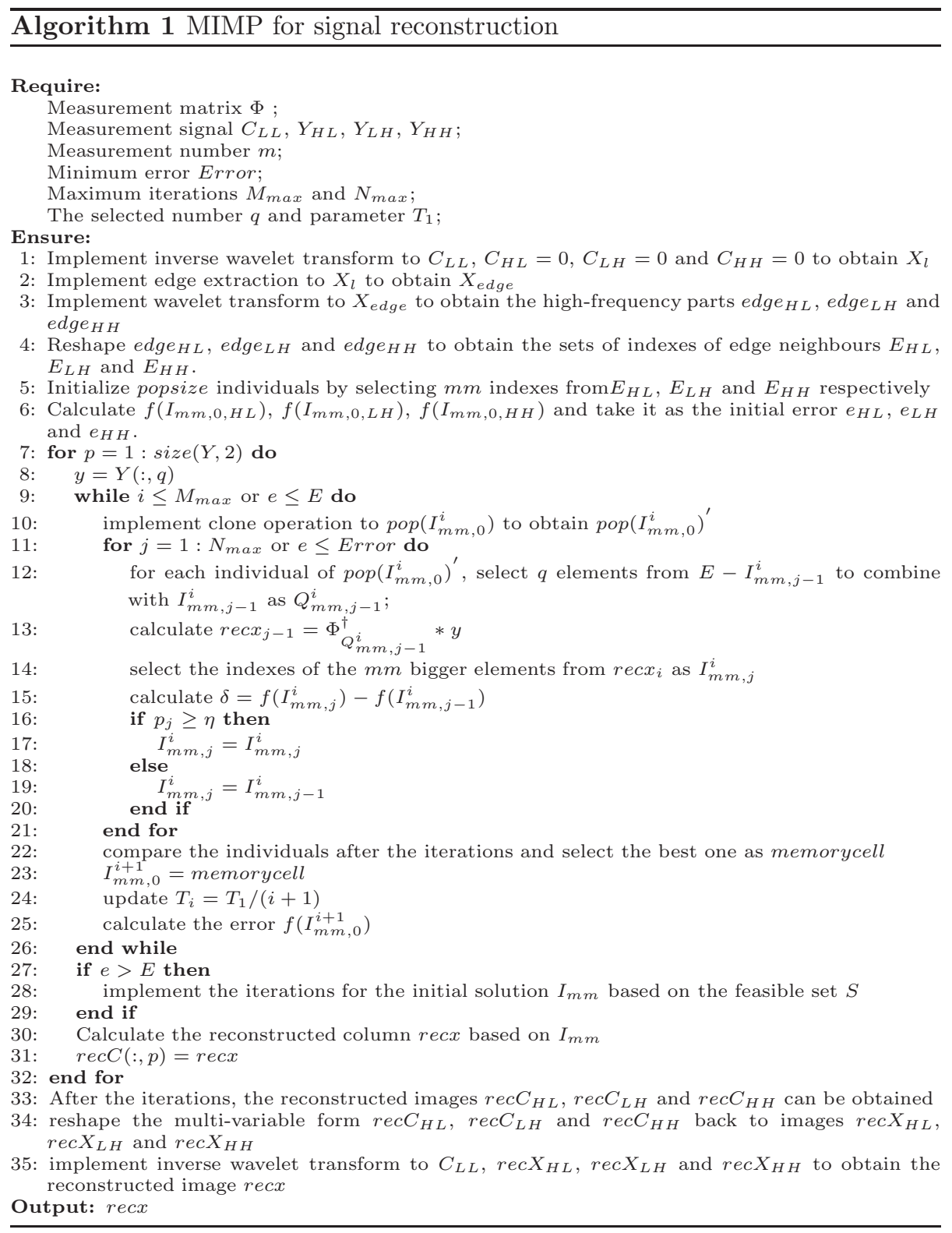


(3) Iteration. The iterations are implemented to reconstruct every variable $x$ of the multi-variable from $X^{\prime}$. As the reconstruction process of $C_{H L}, C_{L H}$ There are three main operations: clone, mutation and selection. Firstly, the clone operation is implemented to the initial individuals, which can generate more individuals to search simultaneously. Secondly, we implement mutation operation, namely the process of the inner loop, to all the individuals. The process of mutation operation for all individuals are the same, so we will take one for example. Firstly, select $q$ elements from $E-I_{m m, j-1}^{i}$ randomly to combine with $I_{m m, j-1}^{i}$ as a new indexes collection $Q_{m m, j-1}^{i}$. Then, calculate $\operatorname{rec}_{m m, j-1}$ by the equation $r e c x_{j-1}=\Phi_{m m, Q_{j-1}^{i}}^{i} * y$ and select the indexes of the larger $m m$ elements from $r e c x_{j}$ as $I_{m m, j}^{i}$. Thirdly, we determine whether the $I_{m m, j}^{i}$ can be chosen as the updated individual based on Metropolis rules. The process of Metropolis rules is: if $p_{j} \geq \eta$, where $p_{j}$ is calculated by $p_{j}=$ $\min \left(1, \exp \left(-\left(f\left(I_{m m, j}^{i}\right)-f\left(I_{m m, j-1}^{i}\right)\right) / T_{i}\right)\right)$ and $\eta$ is a random number chosen in $[0,1]$, implement $I_{m m, j}^{i}=I_{m m, j}^{i}$, otherwise, implement $I_{m m, j}^{i}=I_{m m, j-1}^{i}$. The parameter $T_{i}$ is updated by $T_{i}=T_{1} / \log (i+1)$ in every iteration. Finally, selection can retain the optimal solution to the next iteration.

(4) Terminal condition. The terminal condition of the iterations is that the number of iterations exceeds the maximum number or the error $e$ is smaller than the minimum error Error. If the sets of edge neighbours can not includes all the indexes of the nonzero elements of $E_{H L}, E_{L H}$ and $E_{H H}$, it will lead 270 to a non-accurate reconstruction. However, if the error $e$ can not satisfy the condition $e<$ Error, we implement the above update process to estimate the $I_{m m}$ based on the feasible set $S$.

Finally, the reconstructed multi-variable high-frequency images $\operatorname{rec}_{H L}, \operatorname{rec}_{L H}$ and $\operatorname{rec}_{H H}$ can be obtained through the iterations. Reshape $\operatorname{rec} C_{H L}, \operatorname{rec}_{L H}$

rec $C_{H H}$ to obtain the reconstructed high-frequency images $\operatorname{rec} X_{H L}, \operatorname{rec} X_{L H}$ and $\operatorname{rec} X_{H H}$, then implement inverse wavelet transform to $C_{L L}, \operatorname{rec} X_{H L}, \operatorname{rec} X_{L H}$ and $\operatorname{rec} X_{H H}$ to obtain the reconstructed image $\operatorname{rec} X$.

$l_{0}$ minimization is an NP-hard problem that requires exhaustively listing all 
possibilities of the original signal and has a very high computational complexity.

280 If the sparsity level $K$ is known as a prior, the purpose of signal reconstruction based on $l_{0}$ minimization is to find a global optimal solution in the feasible domain whose size can be calculated by $C_{N}^{K}$. In our proposed method MIMP, we use the edge saliency as the prior knowledge to guide the reconstruction, so the size of the feasible domain can be reduced to $C_{E}^{K}$, where $E$ represents the size of edge collection. Also, the use of multi-variable scheme is beneficial to increase the accuracy of the guidance of the prior knowledge. In addition, based on the optimization function proposed in Theorem 1, the size of the feasible domain can be calculated by $C_{E}^{m m}$, while there are $C_{m m}^{K}$ optimal solutions, which not only can relax the precondition that the sparsity level needs to be known as a prior but also can increase the possibilities to find the optimal solutions. So for many reconstruction problems, our proposed method MIMP has a lower computational complexity than that of solving the original NP-hard problem, and is more likely to find the global optimal solution.

The schematic diagram of MIMP is shown in Figure 2.

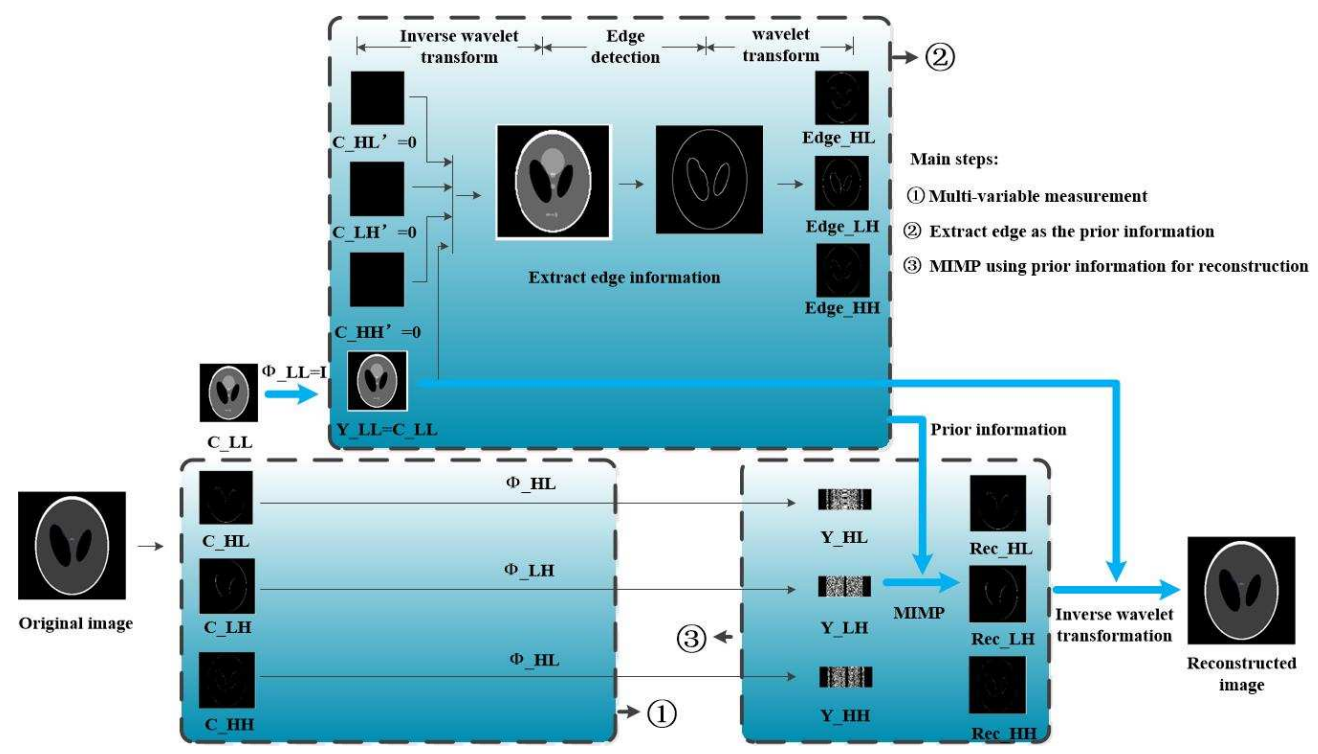

Figure 2: The schematic diagram of image reconstruction based on MIMP 
As shown in Algorithm 1, the main computational complexity of MIMP lies in two parts: the initialization and the iteration. There are two algorithms, i.e. wavelet transform and edge extraction in the process of initialization. The computational complexity of the two algorithms are both upper bounded by $O\left(N^{2}\right)$, where $N$ represents the size of the transformed image. So the computational complexity of initial solution is upper bounded by $4 * O\left(N^{2}\right)$ with 3 times of edge extraction and 1 time of wavelet transformation. In each iteration, the computational complexity lies in step 13 and step 14 shown in Algorithm 1, which both have matrix multiplication. The computational complexity of matrix multiplication is upper bounded by $O\left(N^{3}\right)$. Also, the maximum number of iterations can be calculated by $M_{\max } * N_{\max }$ and there are popsize individuals in the group, so the computation complexity of the iteration is popsize $* M_{\max } N_{\max } O\left(N^{3}\right)$. In conclusion, the computational complexity of MIMP is upper bounded by Equ.(11).

$$
4 * O\left(N^{2}\right)+\text { popsize } * M_{\max } * N_{\max } * O\left(N^{3}\right) .
$$

From Equ.(11), we can see that the computational complexity of MIMP is influenced by the number of iterations $M_{\max } * N_{\max }$ mostly. The clone operation that makes more individuals search simultaneously can reduce the number of iterations. Also, applying the set of edge neighbours as the initial solution is another method to reduce the iterations. Furthermore, some methods can be introduced to optimize the initial solution to reduce the iterations.

\section{Experiment results}

\subsection{Experimental Configuration}

To verify the validity of the proposed method MIMP, compressive sensing reconstruction experiments on several images are conducted. The well-known

320 haar orthogonal wavelet is used for wavelet transformation in the implementation of our algorithm. Neighbourhood structure with the size of $4 * 4$ is used for 
multiple measurement. In the following experiments, a block sampling scheme [44, 45, 46] is utilized to improve the reconstruction speed, that is, the original images are blocked by windows with the size of $32 * 32$. We obtain the measurements from the subblocks and reconstruct these subblocks respectively. The measurement matrix $\phi$ is obtained by extracting $m$ rows of the $N * N$ orthogonal random matrix. We also set the main parameters $M_{\max }=200, N_{\max }=200$, $E=10^{-5}$ in our algorithm for all test images.

All the simulations are implemented in Matlab 2011a on a PC with $3.2 \mathrm{GHz}$ 330 Intel Core $i 5$ processor and $8.0 G B$ memory running the Windows 7 system. Here, the compared algorithms OMP, PFP and BP are implemented using SolveOMP, SolvePFP and SolveBP in SparseLab toolbox [47] respectively.

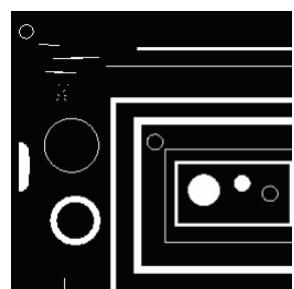

(a)

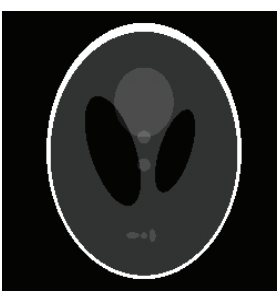

(b)

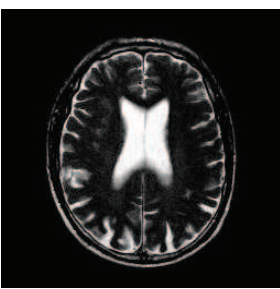

(c)

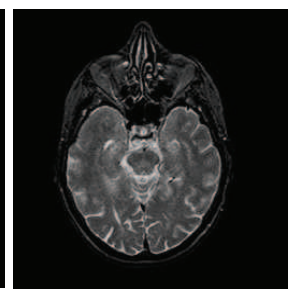

(d)

Figure 3: The test images. (a). blobs image; (b). phantom image; (c). brain MR image; (d). brain CT image

\subsection{Comparison to the state-of-the-art algorithms}

In this experiment, the proposed method MIMP is compared with several state-of-the-art reconstruction algorithms, including multi-variable OMP (MOMP), multi-variable BP (M-BP), multi-variable Polytope Faces Pursuit algorithm (M-PFP), multi-variable compressive sampling orthogonal matching pursuit (M-CoSaOMP) and multi-variable Sparsity Adaptive Matching Pursuit algorithm (M-SAMP), which aims to illustrate the superiority of MIMP by taking advantage of intelligent optimization algorithm in solving $l_{0}$ minimization essentially and introducing the matching strategies of greedy algorithm which performs quite well in reconstruction speed. More specifically, all the compared 
greedy algorithms and the proposed MIMP are based on the multi-variable measurement scheme. The test images are blobs image, phantom image, brain CT image and brain MR image respectively, which are presented in Fig.3. The test images are both famous for their high sparsity level in wavelet domain and obvious edge information. Two indexes, PSNR, which indicates the quality of reconstruction, and runtime, which indicates the reconstruction speed, are adopted to evaluate the proposed method MIMP. The reconstruction of the test images are processed with the measurement rate changing from 0.35 to 0.8 . Experiment results shown in Fig.4, Fig.5, Fig.6 and Fig.7 demonstrate the reconstruction performance comparison between MIMP and the aforementioned algorithms in terms of reconstruction quality (PSNR) and reconstruction speed (Runtime).

From Fig. 4(a), Fig.5 (a), Fig. 6(a) and Fig.7 (a), we can see that the PSNR of image reconstruction gradually increase with the increasing measurement rate in all methods. Obviously, the proposed method MIMP obtains the highest PSNR, i.e., the best reconstruction accuracy, in all the measurement rates. In the case of low measurement rate, MIMP takes on the appearance that PSNR is several $d B$ higher than that of the compared state-of-the-art algorithms. From some cases when the measurement rate is greater than a threshold, the PSNR of MIMP is significantly improved and higher than that of others. As we can see from the figures, the thresholds of blobs image, phantom image, brain CT image, brain MR image are 0.6, 0.5, 0.7 and 0.65 respectively.

365 From the runtime comparison shown in Fig.4 (b), Fig.5 (b), Fig.6 (b) and Fig.7 (b), we can see that the reconstruction speed of the compared stateof-the-art algorithms decrease with the increasing measurement rate but the reconstruction speed of MIMP increases with the increasing measurement rate. We can also see that M-OMP has the fastest reconstruction speed and MIMP 370 is superior to M-BP in almost all the measurement rate. Also, MIMP is faster than M-PFP, M-SAMP and M-CoSaOMP with the measurement rate exceeding 0.75 .

Through the experiment results mentioned above, the reconstruction accu- 


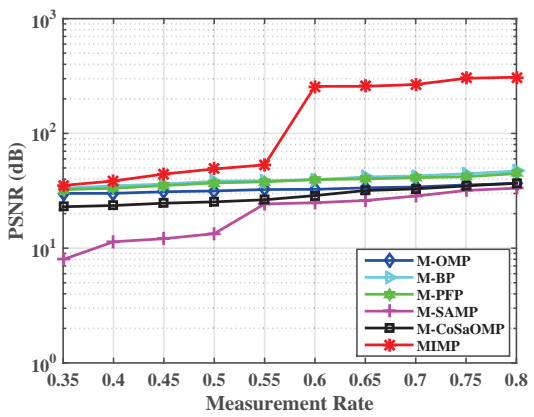

(a)

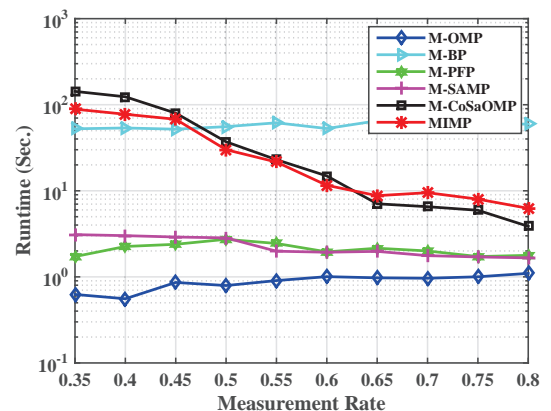

(b)

Figure 4: Reconstruction performance of MIMP, M-OMP, M-PFP, M-SAMP, M$\mathrm{BP}$ and M-CoSaOMP with the measurement rate changing from 0.35 to 0.8 for blobs image. (a). PSNR, (b). Runtime.

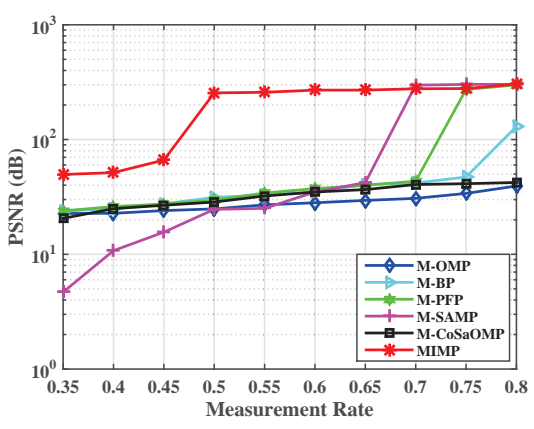

(a)

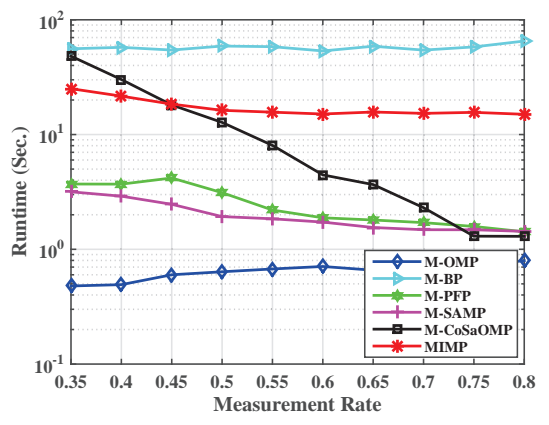

(b)

Figure 5: Reconstruction performance of MIMP, M-OMP, M-PFP, M-SAMP, M$\mathrm{BP}$ and M-CoSaOMP with the measurement rate changing from 0.35 to 0.8 for phantom image. (a). PSNR, (b). Runtime. 


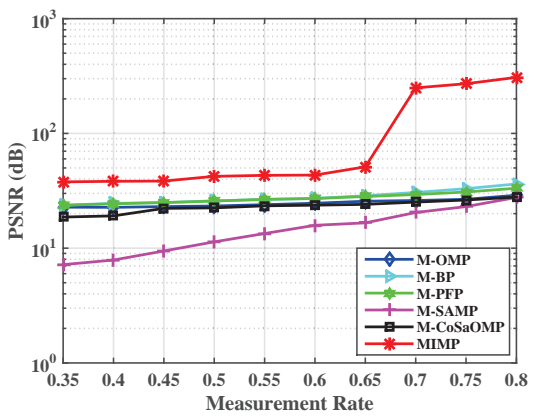

(a)

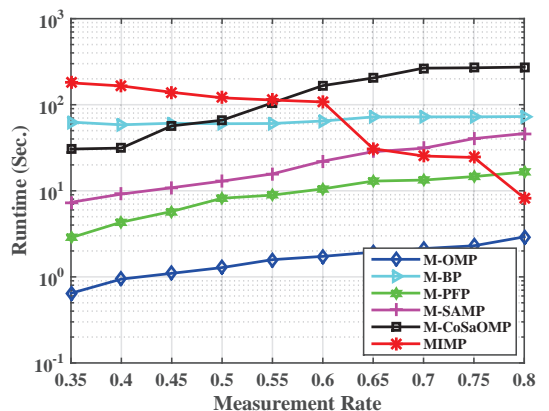

(b)

Figure 6: Reconstruction performance of MIMP, M-OMP, M-PFP, M-SAMP, M$\mathrm{BP}$ and M-CoSaOMP with the measurement rate changing from 0.35 to 0.8 for brain MR image. (a). PSNR, (b). Runtime.

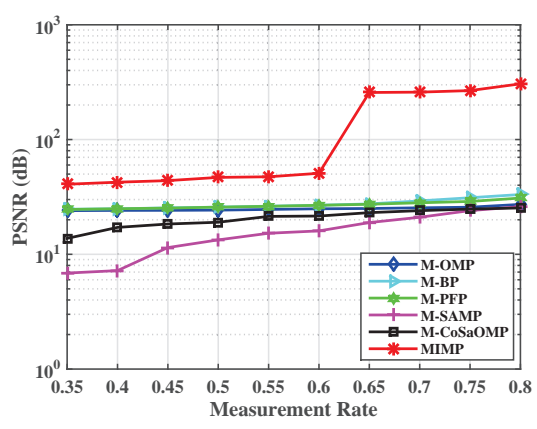

(a)

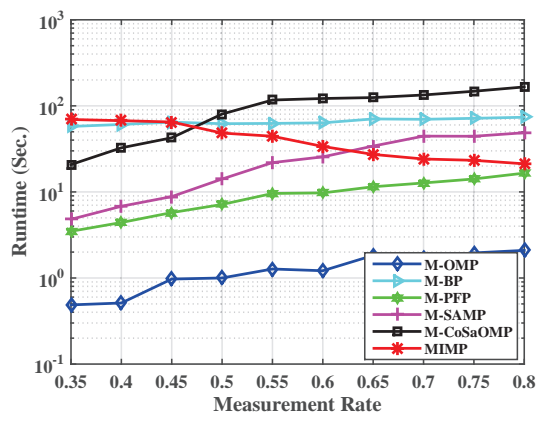

(b)

Figure 7: Reconstruction performance of MIMP, M-OMP, M-PFP, M-SAMP, M$\mathrm{BP}$ and M-CoSaOMP with the measurement rate changing from 0.35 to 0.8 for brain CT image. (a). PSNR, (b). Runtime. 
racy of MIMP is superior to the compared state-of-the-art algorithms. As we use multi-variable measurement scheme in all the methods, the main reason of the better performance of MIMP is that we take the advantage of the intelligent optimization algorithm in solving combinatorial optimization problems and searching the global optimal solution. Also, we design novel and effective updating mechanism by introducing the matching strategies of greedy algorithm to make MIMP more likely to find the optimal solution. More specifically, MIMP improves the performance of image reconstruction by means of solving $l_{0}$ minimization essentially and searching the global optimal solution more likely.

The experiment results also demonstrate that the reconstruction speed of MIMP is faster than M-BP but slower than the other compared algorithms in many cases. Compared with many state-of-the-art greedy algorithms, MIMP slows down due to the global searching, which needs more iterations to find the global optimal solution. However, with the measurement rate increasing, the performance of the updating mechanism of MIMP becomes more effective, which can reduce the number of iteration to accelerate the reconstruction speed. In addition, the using of prior knowledge can also improve the reconstruction speed through reducing the computational complexity of MIMP, which makes the reconstruction speed of MIMP more reasonable.

\subsection{Comparison to algorithms using prior knowledge}

In this experiment, the proposed method MIMP is compared with some state-of-the-art compressive sensing reconstruction methods which use the multivariable scheme and set some structures of images as the prior knowledge for image reconstruction, which aims to illustrate the superiority of MIMP by using the multi-variable scheme and utilizing the edge saliency as the prior knowledge. The structured based reconstruction methods are well known Tree-CoSaMP, TSWCS, edge based multi-variable pursuit algorithm (EMPA), edge guided compressive sensing (EdgeCS), multi-variable pursuit algorithm using multivariable $K$ distribution (MPA-MK) and multi-variable pursuit algorithm using multi-variable Laplace distribution (MPA-ML) respectively. Note that the Tree- 
Table 1: Reconstruction performance of Tree-CoSaMP, TSWCS, EMPA, EdgeCS, MPA-MK, MPA-ML and MIMP for blobs image and phantom image with the measurement rate fixed as 0.4. The evaluate indexes are PSNR (dB), SSIM and Time (Sec.).

\begin{tabular}{lcccc}
\hline Image & Algorithms & PSNR $(\mathrm{dB})$ & SSIM & Time (Sec.) \\
\hline \multirow{4}{*}{ blobs } & Tree-CoSaMP & 37.0963 & $\mathbf{1 . 0 0 0 0}$ & 521.0282 \\
& TSWCS & $\mathbf{1 6 9 . 8 7 7 4}$ & $\mathbf{1 . 0 0 0 0}$ & 801.3333 \\
& EMPA & 41.8219 & 0.9997 & 3.8839 \\
& EdgeCS & 64.9615 & $\mathbf{1 . 0 0 0 0}$ & 1.0586 \\
& MPA-MK & 33.4425 & 0.9983 & $\mathbf{0 . 5 6 3 7}$ \\
& MPA-ML & 33.2983 & 0.9983 & 0.5924 \\
MIMP & 48.5922 & $\mathbf{1 . 0 0 0 0}$ & 30.0091 \\
& & & & \\
Tree-CoSaMP & 41.7257 & 0.9965 & 843.1793 \\
TSWCS & 126.9542 & $\mathbf{1 . 0 0 0 0}$ & 1046.0712 \\
& EMPA & 39.2611 & 0.9867 & 3.8156 \\
EdgeCS & 108.0631 & $\mathbf{1 . 0 0 0 0}$ & 0.8732 \\
MPA-MK & 31.4908 & 0.9870 & $\mathbf{0 . 5 6 6 9}$ \\
MPA-ML & 31.4896 & 0.9870 & 0.5693 \\
MIMP & $\mathbf{2 5 4 . 9 7 8 9}$ & $\mathbf{1 . 0 0 0 0}$ & 16.2553 \\
\hline
\end{tabular}

CoSaMP and TSWCS exploit the tree structure of wavelet coefficients and they

405 are very effective in image reconstruction, especially in areas of image structures. EMPA and EdgeCS use the edge information as the prior knowledge and produce state-of-the-art reconstruction quality. MPA-MK and MPA-ML are multi-variable pursuit algorithms based on multi-variable measurement scheme using multi-variable scale mixture models as the prior distributions, which provide superior performance compared with many state-of-the-art compressive sensing reconstruction algorithms. The test images are blobs image, phantom image, brain CT image, brain MR image and liver CT image respectively, where the size of liver CT image is $512 * 512$ and that of others are $256 * 256$. Another quantitative measure, Structural Similarity (SSIM) index measurement [48], is adopted to the objective evaluation. A SSIM value much closer to 1 indicates the structure of the reconstructed image is more similar to the original image. The measurement rate is fixed as 0.4 for sparse images and 0.5 for medical images. Table 1 shows the PSNR, SSIM and Runtime comparison between the proposed method MIMP and Tree-CoSaMP, TSWCS, EMPA, EdgeCS, MPA-

420 MK and MPA-ML for sparse image reconstruction. Experiment results shown in Table 2 demonstrate the superior reconstruction performance of our pro- 
posed method MIMP by the comparison with Tree-CoSaMP, TSWCS, EMPA, EdgeCS, MPA-MK and MPA-ML for medical image reconstruction in terms of PSNR, SSIM and Runtime. The reconstructed images and their corresponding magnified parts based on the aforementioned methods are shown in Fig.8, Fig.9 and Fig.10, which aims to illustrate the superior performance of MIMP in reconstruction visual.

Table 2: Reconstruction performance of Tree-CoSaMP, TSWCS, EMPA, EdgeCS, MPA-MK, MPA-ML and MIMP for brain MR image, brain CT image and liver CT image with the measurement rate fixed as 0.5. The evaluate indexes are PSNR (dB), SSIM and Time (Sec.).

\begin{tabular}{lcccc}
\hline Image & Algorithms & PSNR $(\mathrm{dB})$ & SSIM & Time (Sec.) \\
\hline & Tree-CoSaMP & 31.7627 & 0.8621 & 424.5496 \\
& TSWCS & 26.0477 & 0.7522 & 891.328 \\
brain MR & EMPA & 29.7933 & 0.8845 & 3.3043 \\
& EdgeCS & 36.0830 & 0.9750 & 6.0909 \\
& MPA-MK & 28.7693 & 0.9528 & $\mathbf{0 . 5 8 0 0}$ \\
& MPA-ML & 27.9408 & 0.9392 & 0.6024 \\
& MIMP & $\mathbf{4 6 . 7 8 2 9}$ & $\mathbf{0 . 9 9 7 7}$ & 48.2934 \\
& & & & \\
brain CT & Tree-CoSaMP & 28.0566 & 0.8621 & 649.3453 \\
& TSWCS & 27.2981 & 0.8561 & 874.0045 \\
& EMPA & 30.9356 & 0.9035 & 3.3236 \\
& EdgeCS & 35.7729 & 0.9701 & 5.9105 \\
& MPA-MK & 27.9925 & 0.9328 & $\mathbf{0 . 5 9 2 2}$ \\
& MPA-ML & 27.3202 & 0.9229 & 0.6131 \\
& MIMP & $\mathbf{4 2 . 2 8 1 7}$ & $\mathbf{0 . 9 9 3 6}$ & 120.2910 \\
& & & \\
& Tree-CoSaMP & 28.0207 & 0.9655 & 1486.2143 \\
& TSWCS & 32.9495 & 0.9832 & 3428.6175 \\
& EMPA & 35.0608 & $\mathbf{1 . 0 0 0 0}$ & 8.8397 \\
& EdgeCS & 36.6095 & 0.7308 & 26.8655 \\
& MPA-MK & 35.1983 & 0.9997 & 1.6344 \\
& MPA-ML & 34.2068 & 0.9997 & $\mathbf{1 . 5 7 0 3}$ \\
& MIMP & $\mathbf{3 8 . 6 5 4 1}$ & $\mathbf{1 . 0 0 0 0}$ & 334.1973 \\
\hline
\end{tabular}

From Table 1, we can see that the proposed method MIMP obtains the highest PSNR and SSIM in phantom image reconstruction. In the case of blobs image reconstruction, MIMP is superior to Tree-CoSaMP, EMPA, MPA-MK and MPA-ML on reconstruction quality. Table 2 shows that the proposed method MIMP outperforms other competing methods both on PSNR and SSIM for all the medical image reconstruction. As shown in Table 1 and Table 2, for all the test images, the reconstruction speed of MIMP is faster than that of Tree- 
CoSaMP and TSWCS but slower than that of EMPA, EgdeCS, MPA-MK and MPA-ML.

The experiment results demonstrate that MIMP improves the PSNR and SSIM of sparse image reconstruction significantly and the advantage PSNR gain of MIMP over the competing methods for medical image reconstruction is up to several $d B$. The main reason is that phantom image has a higher sparsity in the wavelet domain and the edge saliency obtained from the low-frequency part of wavelet coefficients has a strong guidance for the process of reconstruction. Although the guidance of edge information of brain MR image, brain CT image and liver CT image are not that strong like that of phantom image, it can also improve the reconstruction accuracy and reconstruction speed. Also, the multivariable measurement scheme and multi-variable joint reconstruction can not only increase the guidance accuracy of the prior knowledge for reconstruction but also greatly accelerate the reconstruction speed.

To demonstrate the superiority of the proposed method MIMP over other compared methods clearly, the visual quality of the reconstructed images based on the test methods are shown in Fig.8, Fig.9 and Fig.10. The odd rows present the reconstructed images and the even rows represent the local enlarged parts marked with the red blocks corresponding to the reconstructed images, in particular the areas of edges. From Fig.8, Fig.9 and Fig.10, we can observe that the Tree-CoSaMP, EMPA, EdgeCS, MPA-MK and MPA-ML tend to blur the reconstructed images, especially in the edge region. Although TSWCS method has a high PSNR for blobs image and phantom image, it generates artefacts in the areas of edges. In contrast, the proposed method MIMP performs better on both the edge region and the smooth region of the reconstructed images.

460 Especially from the local enlarged parts of the reconstructed images, we can see that MIMP provides the reconstruction results with a best visual. 

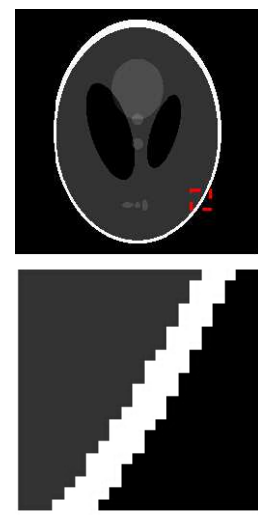

(a). Original
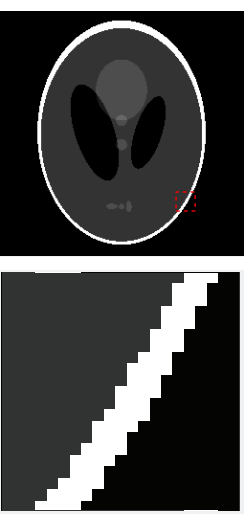

(e). EdgeCS

$\mathrm{PSNR}=108.0631$ $\mathrm{SSIM}=1.0000$
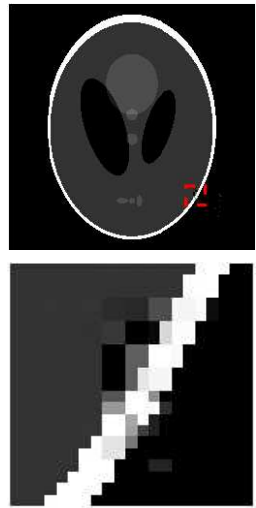

(b). Tree-CoSaMP $\mathrm{PSNR}=41.7257$ $\mathrm{SSIM}=0.9965$
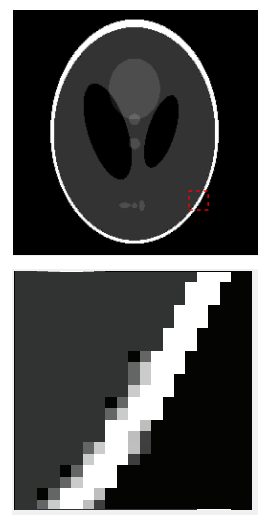

(f). MPA-MK

$\mathrm{PSNR}=31.4908$ $\mathrm{SSIM}=0.9870$
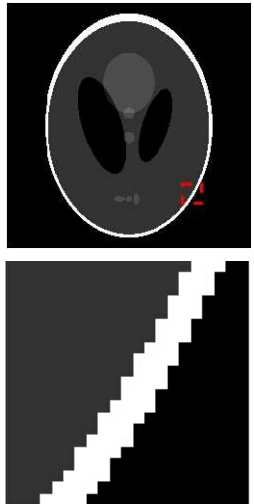

(c). TSWCS

$\mathrm{PSNR}=126.9542$ $\mathrm{SSIM}=1.0000$
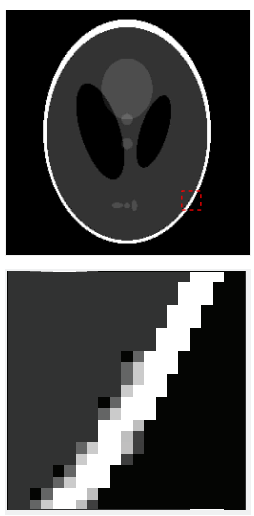

(g). MPA-ML

$\mathrm{PSNR}=31.4896$ $\mathrm{SSIM}=0.9870$
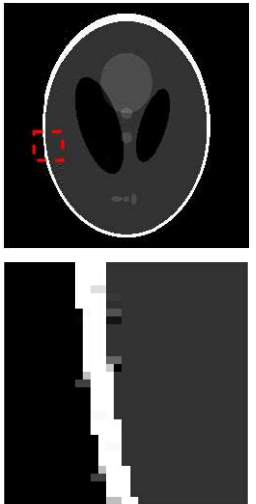

(d). EMPA

$\mathrm{PSNR}=39.2611$

$\mathrm{SSIM}=0.9867$
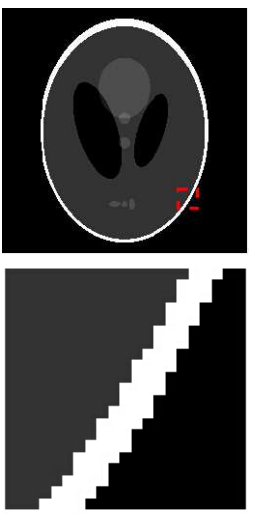

(h). MIMP

$\mathrm{PSNR}=254.9789$

$\mathrm{SSIM}=1.0000$

Figure 8: Visual performance of Tree-CoSaMP, TSWCS, EMPA, EdgeCS, MPAMK, MPA-ML and MIMP for phantom image when the measurement rate is fixed as 0.4 . The odd rows represent the global reconstructed images. The even rows represent the local enlarged parts marked by the red blocks. 

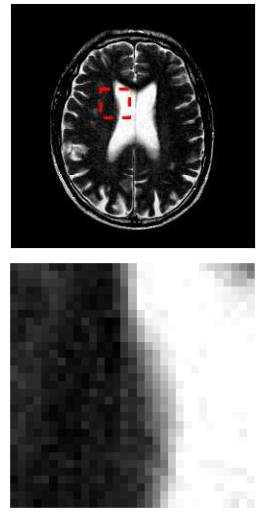

(a). Original
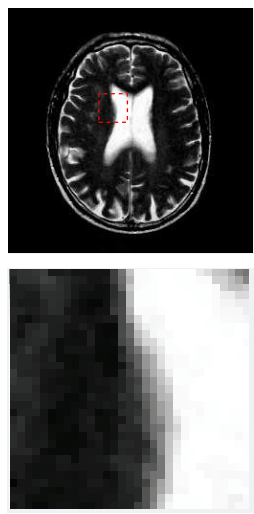

(e). EdgeCS

$\mathrm{PSNR}=36.0830$

$\mathrm{SSIM}=0.9750$
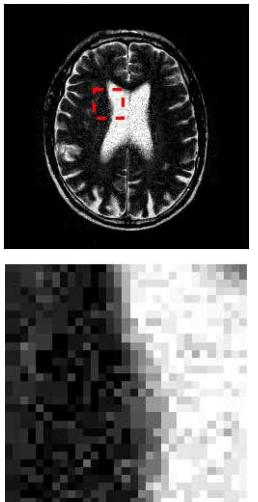

(b). Tree-CoSaMP $\mathrm{PSNR}=31.7627$

$\mathrm{SSIM}=0.8621$
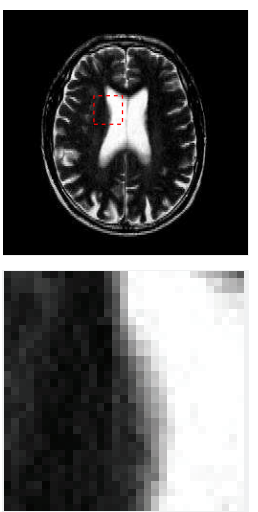

(f). MPA-MK

$\mathrm{PSNR}=28.7693$ SSIM $=0.9528$
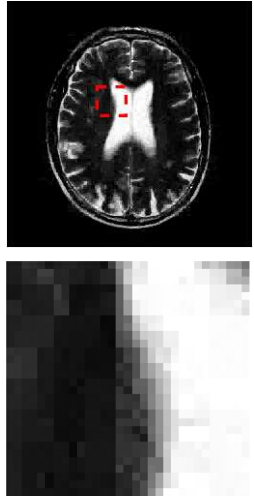

(c). TSWCS

$\mathrm{PSNR}=26.0477$

$\mathrm{SSIM}=0.7522$
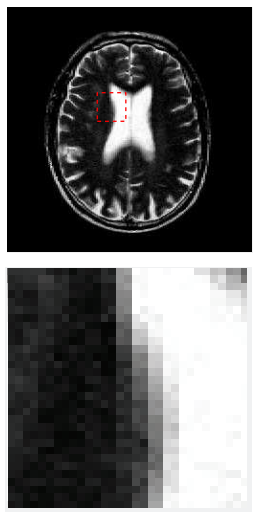

(g). MPA-ML

$\mathrm{PSNR}=27.9408$

SSIM $=0.9392$
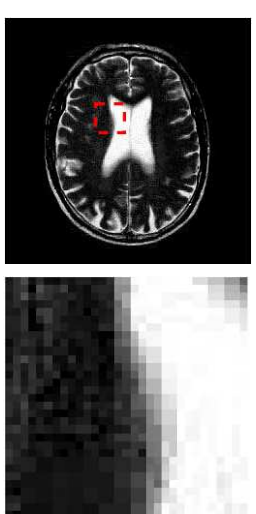

(d). EMPA

$\mathrm{PSNR}=29.7933$

$\mathrm{SSIM}=0.8845$
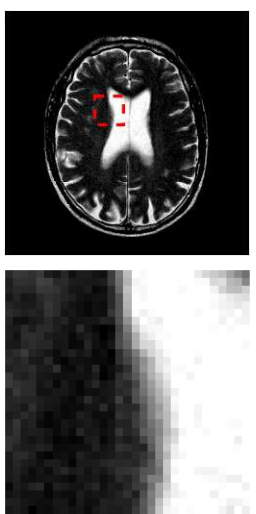

(h). MIMP

$\mathrm{PSNR}=46.7829$

$\mathrm{SSIM}=0.9977$

Figure 9: Visual performance of Tree-CoSaMP, TSWCS, EMPA, EdgeCS, MPAMK, MPA-ML and MIMP for brain MRI image when the measurement rate is fixed as 0.5 . The odd rows represent the global reconstructed images. The even rows represent the local enlarged parts marked by the red blocks. 


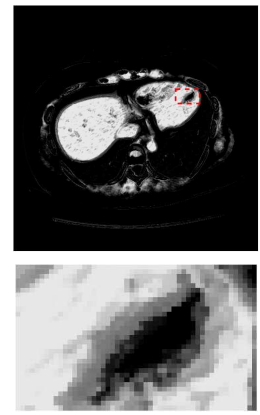

(a). Original
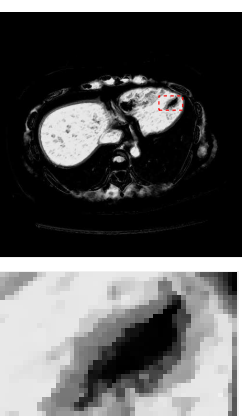

(e). EdgeCS

$\mathrm{PSNR}=36.6095$

$\mathrm{SSIM}=0.9308$
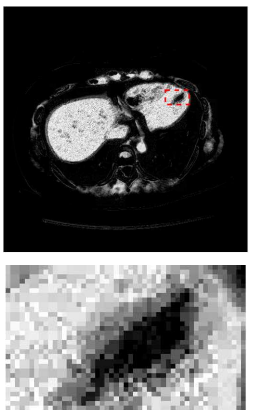

(b). Tree-CoSaMP $\mathrm{PSNR}=28.0207$ $\mathrm{SSIM}=0.9655$
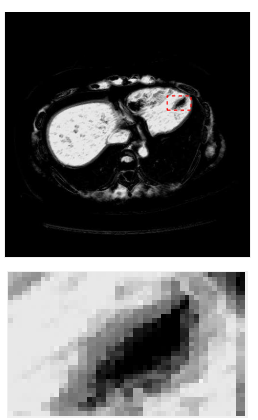

(f). MPA-MK

$\mathrm{PSNR}=35.1983$

$\mathrm{SSIM}=0.9997$
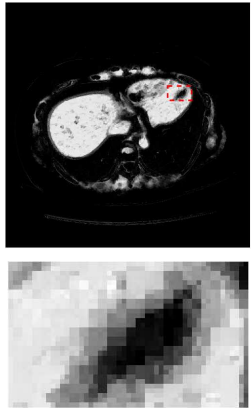

(c). TSWCS

$\mathrm{PSNR}=32.9495$

$\mathrm{SSIM}=0.9832$
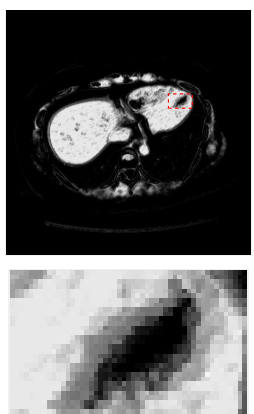

(g). MPA-ML

$\mathrm{PSNR}=34.2068$

$\mathrm{SSIM}=0.9997$
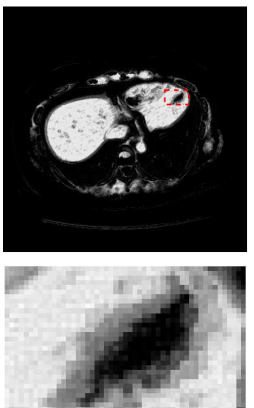

(d). EMPA

$\mathrm{PSNR}=35.0608$

$\mathrm{SSIM}=1.0000$
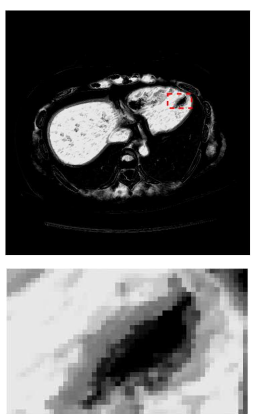

(h). MIMP

$\mathrm{PSNR}=38.6541$

$\mathrm{SSIM}=1.0000$

Figure 10: Visual performance of Tree-CoSaMP, TSWCS, EMPA, EdgeCS, MPAMK, MPA-ML and MIMP for liver CT image when the measurement rate is fixed as 0.5. The odd rows represent the global reconstructed images. The even rows represent the local enlarged parts marked by the red blocks. 


\section{Conclusion}

This paper proposes a novel method multi-variable intelligent matching pursuit algorithm (MIMP) using prior knowledge for image reconstruction with the

465 sparsity level unknown as a prior. First of all, the proposed method MIMP can solve $l_{0}$ minimization problem essentially and find the global optimal solution by taking advantage of intelligent optimization algorithm which is famous for its superior performance in solving combinatorial optimization problems and searching the global optimal solution. MIMP has a reasonable relatively faster reconstruction speed by introducing the matching strategies of greedy algorithm to design the updating mechanism. Also, the multi-variable scheme is used to sample the original image and joint reconstruction is implemented for the measurements, which contribute a lot to enhancing the reconstruction performance. In addition, under the multi-variable measurement scheme, the edge saliency 475 can be obtained from the low frequency wavelet coefficients and set as the prior knowledge to guide the high frequency wavelet coefficients reconstruction, which can not only reduce the computational complexity but also improve the reconstruction quality. Experiment results on several images demonstrate that MIMP outperforms the state-of-the-art algorithms such as multi-variable OMP 480 (M-OMP), multi-variable PFP (M-PFP), multi-variable (SAMP), multi-variable BP (M-BP), multi-variable CoSaOMP (M-CoSaMP) and structure based algorithms such as tree-CoSaMP, TSWCS, EMPA EdgeCS, MPA-MK and MPAML, especially in the areas of edges. Through the experiment results, it can be demonstrated that our proposed method MIMP significantly improves the ${ }_{485}$ reconstruction quality of those images with the obvious edges and high sparsity in wavelet domain, such as CT images and MR images. Nevertheless, the proposed method MIMP also has room for improvement. One possible extension of our work would be to introduce more effective updating mechanism into MIMP to further reduce the computational complexity. Another interesting challenge would be to introduce more structures of image as the prior knowledge, such as texture, which can guide the process of MIMP for image reconstruction. 


\section{ACKNOWLEDGMENT}

This work is financially supported by National Science Foundations of China (No.61174016) and (No.61171197).

\section{References}

[1] D. L. Donoho, Compressed sensing, IEEE Transactions on Information Theory 52 (4) (2006) 1289-1306.

[2] E. J. Candès, M. B. Wakin, An introduction to compressive sampling, IEEE Signal Processing Magazine 25 (2) (2008) 21-30.

[3] E. J. Candès, J. Romberg, T. Tao, Robust uncertainty principles: Exact signal reconstruction from highly incomplete frequency information, IEEE Transactions on Information Theory 52 (2) (2006) 489-509.

[4] P.-Y. Chen, I. W. Selesnick, Group-sparse signal denoising: non-convex regularization, convex optimization, IEEE Transactions on Signal Processing 62 (13) (2014) 3464-3478.

[5] D. L. Donoho, M. Elad, On the stability of the basis pursuit in the presence of noise, Signal Processing 86 (3) (2006) 511-532.

[6] S. S. Chen, D. L. Donoho, M. A. Saunders, Atomic decomposition by basis pursuit, SIAM journal on scientific computing 20 (1) (1998) 33-61.

[7] W. Dai, O. Milenkovic, Subspace pursuit for compressive sensing signal reconstruction, IEEE Transactions on Information Theory 55 (5) (2009) 2230-2249.

[8] Z. Liu, Z. Wei, W. Sun, An iteratively approximated gradient projection algorithm for sparse signal reconstruction, Applied Mathematics and Computation 228 (2014) 454-462. 
[9] G. Chen, D. Li, J. Zhang, Iterative gradient projection algorithm for twodimensional compressive sensing sparse image reconstruction, Signal Processing 104 (2014) 15-26.

[10] L. B. Montefusco, D. Lazzaro, S. Papi, Nonlinear filtering for sparse signal recovery from incomplete measurements, IEEE Transactions on Signal Processing 57 (7) (2009) 2494-2502.

[11] T. Blumensath, M. E. Davies, Iterative hard thresholding for compressed sensing, Applied and Computational Harmonic Analysis 27 (3) (2009) 265274 .

[12] D. L. Donoho, Y. Tsaig, I. Drori, J.-L. Starck, Sparse solution of underdetermined systems of linear equations by stagewise orthogonal matching pursuit, IEEE Transactions on Information Theory 58 (2) (2012) 10941121.

[13] J. Tropp, A. C. Gilbert, et al., Signal recovery from random measurements via orthogonal matching pursuit, IEEE Transactions on Information Theory 53 (12) (2007) 4655-4666.

[14] M. Masood, T. Y. Al-Naffouri, Sparse reconstruction using distribution agnostic bayesian matching pursuit, IEEE Transactions on Signal Processing 61 (21) (2013) 5298-5309.

535 [15] M. Tuba, N. Bacanin, Improved seeker optimization algorithm hybridized with firefly algorithm for constrained optimization problems, Neurocomputing 143 (2014) 197-207.

[16] H. Che, C. Li, X. He, T. Huang, An intelligent method of swarm neural networks for equalities-constrained nonconvex optimization, Neurocomputing 167 (2015) 569-577.

[17] X. Du, L. Cheng, G. Cheng, A heuristic search algorithm for the multiple measurement vectors problem, Signal Processing 100 (2014) 1-8. 
[18] X. Du, L. Cheng, D. Chen, A simulated annealing algorithm for sparse recovery by 10 minimization, Neurocomputing 131 (2014) 98-104.

[19] D. Li, M. Li, Y. Shen, Y. Wang, Q. Wang, Ga-bfo based signal reconstruction for compressive sensing, in: 2013 IEEE International Conference on Information and Automation (ICIA), 2013, pp. 1023-1028.

[20] D. Li, C. Shi, Q. Wang, Y. Shen, Y. Wang, Artificial immune algorithm based signal reconstruction for compressive sensing, in: 2014 IEEE International conference on Instrumentation and Measurement Technology Conference (I2MTC) Proceedings, 2014, pp. 76-81.

[21] Y. Zhong, L. Zhang, An adaptive artificial immune network for supervised classification of multi-/hyperspectral remote sensing imagery, IEEE Transactions on Geoscience and Remote Sensing 50 (3) (2012) 894-909.

[22] H. Jiao, Y. Zhong, L. Zhang, Artificial dna computing-based spectral encoding and matching algorithm for hyperspectral remote sensing data, IEEE Transactions on Geoscience and Remote Sensing 50 (10) (2012) 40854104 .

[23] S. Jacob, M. S. Nair, Image reconstruction from random samples using multiscale regression framework, Neurocomputing.

[24] M. Afonso, J. M. Sanches, Image reconstruction under multiplicative speckle noise using total variation, Neurocomputing 150 (2015) 200-213.

[25] J. Xiao, M. K.-P. Ng, Y.-F. Yang, On the convergence of nonconvex minimization methods for image recovery, IEEE Transactions on Image Processing 24 (5) (2015) 1587-1598.

[26] T. Z. Teisseyre, J. L. Paulsen, V. S. Bajaj, N. W. Halpern-Manners, A. Pines, Compressive sampling with prior information in remotely detected mri of microfluidic devices, Journal of Magnetic Resonance 216 (2012) $13-20$. 
[27] A. Majumdar, R. K. Ward, Compressive color imaging with group-sparsity on analysis prior, in: 2010 17th IEEE International Conference on Image Processing (ICIP), 2010, pp. 1337-1340.

[28] C. La, M. N. Do, Tree-based orthogonal matching pursuit algorithm for signal reconstruction, in: 2006 IEEE International Conference on Image Processing, 2006, pp. 1277-1280.

[29] M. F. Duarte, M. B. Wakin, R. G. Baraniuk, Wavelet-domain compressive signal reconstruction using a hidden markov tree model, in: 2008 IEEE International Conference on Acoustics, Speech and Signal Processing, 2008. ICASSP, 2008, pp. 5137-5140.

[30] M. N. Do, C. N. La, Tree-based majorize-maximize algorithm for compressed sensing with sparse-tree prior, in: 2007. 2nd IEEE International Workshop on Computational Advances in Multi-Sensor Adaptive Processing, 2007. CAMPSAP, 2007, pp. 129-132.

[31] J. Wu, F. Liu, L. Jiao, X. Wang, B. Hou, Multivariate compressive sensing for image reconstruction in the wavelet domain: using scale mixture models, IEEE Transactions on Image Processing 20 (12) (2011) 3483-3494.

[32] N. S. Rao, R. D. Nowak, S. J. Wright, N. G. Kingsbury, Convex approaches to model wavelet sparsity patterns, in: 2011 18th IEEE International Conference on Image Processing (ICIP), 2011, pp. 1917-1920.

[33] L. He, L. Carin, Exploiting structure in wavelet-based bayesian compressive sensing, IEEE Transactions on Signal Processing 57 (9) (2009) 3488-3497.

[34] E. Vera, L. Mancera, S. D. Babacan, R. Molina, A. K. Katsaggelos, Bayesian compressive sensing of wavelet coefficients using multiscale laplacian priors, in: IEEE/SP 15th Workshop on Statistical Signal Processing, 2009, pp. 229-232. 
[35] S. Som, P. Schniter, Compressive imaging using approximate message passing and a markov-tree prior, IEEE Transactions on Signal Processing 60 (7) (2012) 3439-3448.

[36] W. Guo, W. Yin, Edgecs: Edge guided compressive sensing reconstruction, in: Visual Communications and Image Processing 2010, 2010, pp. 77440L77440L.

[37] J. Wu, F. Liu, L. Jiao, X. Wang, Multivariate pursuit image reconstruction using prior information beyond sparsity, Signal Processing 93 (6) (2013) $1662-1672$.

[38] Y. Tsaig, D. L. Donoho, Extensions of compressed sensing, Signal processing 86 (3) (2006) 549-571.

[39] M. D. Plumbley, Recovery of sparse representations by polytope faces pursuit, in: Independent Component Analysis and Blind Signal Separation, Springer, 2006, pp. 206-213.

[40] H. Huang, A. Makur, Backtracking-based matching pursuit method for sparse signal reconstruction, IEEE Signal Processing Letters 18 (7) (2011) 391-394.

[41] D. Needell, J. A. Tropp, Cosamp: Iterative signal recovery from incomplete and inaccurate samples, Applied and Computational Harmonic Analysis 26 (3) (2009) 301-321.

[42] T. T. Do, L. Gan, N. Nguyen, T. D. Tran, Sparsity adaptive matching pursuit algorithm for practical compressed sensing, in: 2008 42nd Asilomar Conference on Signals, Systems and Computers, 2008, pp. 581-587.

[43] R. G. Baraniuk, V. Cevher, M. F. Duarte, C. Hegde, Model-based compressive sensing, IEEE Transactions on Information Theory 56 (4) (2010) 1982-2001. 
[44] M. Stojnic, F. Parvaresh, B. Hassibi, On the reconstruction of block-sparse signals with an optimal number of measurements, IEEE Transactions on Signal Processing 57 (8) (2009) 3075-3085.

[45] J. K. Pant, W.-S. Lu, A. Antoniou, Reconstruction of block-sparse signals by using an $12 /$ p-regularized least-squares algorithm, in: 2012 IEEE International Symposium on Circuits and Systems (ISCAS), 2012, pp. 277-280.

[46] J. J. Zhao, J. X. Sun, S. L. Zhou, L. Hu, Imaging of transmission equipment based on block compressed sensing, in: Applied Mechanics and Materials, Vol. 190, 2012, pp. 998-1001.

[47] D. Needell, Topics in compressed sensing, arXiv preprint arXiv:0905.4482.

[48] Z. Wang, A. C. Bovik, H. R. Sheikh, E. P. Simoncelli, Image quality assessment: from error visibility to structural similarity, IEEE Transaction s onImage Processing 13 (4) (2004) 600-612. 